\title{
Open Problem 3: On the Integrability of Hamiltonian Systems
}

\section{Carles Simó $^{1}$}

Assume that a complex analytical Hamiltonian system has a solution $\gamma(t)$ and that the Galois groups associated to the $k$-th variational equations along $\gamma(t)$ (see [1]) have identity components which are abelian for all $k$.

But assume also that the system is not integrable, with meromorphic first integrals, in the Liouville-Arnold sense.

How one can characterize those systems? Do there exist some formal integrals in a vicinity of $\gamma(t)$ ?

See [1] for background and, in special, section 7 for related open questions.

\section{Reference}

1. Morales, J.J., Ramis, J.P., Simó, C.: Integrability of Hamiltonian systems and differential Galois groups of higher variational equations. Annales Sci. de l'ENS $4^{e}$ série 40, 845-884 (2007)

$凶$ Carles Simó

carles@maia.ub.es

1 Departament de Matemàtica Aplicada i Anàlisi, Universitat de Barcelona, Gran Via 585, 08007 Barcelona, Spain 